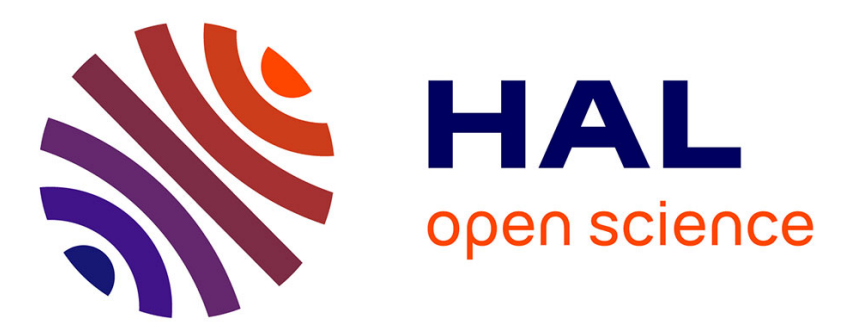

\title{
Pressure-Induced Structural Transition in LuFe2O4: Towards a New Charge Ordered State
}

\author{
Jérôme Rouquette, Julien Haines, Ali Al-Zein, Philippe Papet, Françoise \\ Damay, Julie Bourgeois, Tahar Hammouda, Fabrice Doré, Antoine Maignan, \\ Maryvonne Hervieu, et al.
}

\section{To cite this version:}

Jérôme Rouquette, Julien Haines, Ali Al-Zein, Philippe Papet, Françoise Damay, et al.. PressureInduced Structural Transition in LuFe2O4: Towards a New Charge Ordered State. Physical Review Letters, 2010, 105 (23), pp.237203. 10.1103/PhysRevLett.105.237203 . hal-00545406

\section{HAL Id: hal-00545406 https://hal.science/hal-00545406}

Submitted on 10 Dec 2010

HAL is a multi-disciplinary open access archive for the deposit and dissemination of scientific research documents, whether they are published or not. The documents may come from teaching and research institutions in France or abroad, or from public or private research centers.
L'archive ouverte pluridisciplinaire HAL, est destinée au dépôt et à la diffusion de documents scientifiques de niveau recherche, publiés ou non, émanant des établissements d'enseignement et de recherche français ou étrangers, des laboratoires publics ou privés. 


\title{
Pressure Induced Structural Transition in $\mathrm{LuFe}_{2} \mathrm{O}_{4}$ : Towards a New Charge Ordered State
}

\author{
J. Rouquette ${ }^{1 *}$, J. Haines ${ }^{1}$, A. Al-Zein ${ }^{1,2}$, Ph. Papet ${ }^{1}$, F. Damay ${ }^{3}$, J. Bourgeois ${ }^{3,4}$, T. Hammouda ${ }^{5}$, \\ A. Maignan ${ }^{4}$, M. Hervieu ${ }^{4}$, C. Martin ${ }^{4}$ \\ ${ }^{1}$ Institut Charles Gerhardt UMR CNRS 5253, Équipe Chimie et Cristallochimie des Matériaux (C2M), \\ Université Montpellier II, Place Eugène Bataillon, cc1503, 34095 Montpellier cedex 5, France. \\ ${ }^{2}$ LCVN, UMR CNRS 558\%, Université Montpellier II, 34095 Montpellier, France. \\ ${ }^{3}$ Laboratoire Léon Brillouin, UMR 12, CEA-Saclay, CEA-CNRS, 91191 Gif-sur-Yvette, France. \\ ${ }^{4}$ Laboratoire CRISMAT, ENSICAEN, UMR 6508 CNRS, Caen, France. and \\ ${ }^{5}$ Clermont Université, Université Blaise Pascal, Laboratoire Magmas et Volcans, CNRS UMR 6524, France.*
}

\begin{abstract}
The electronic ferroelectric lutetium ferrite $\left(\mathrm{LuFe}_{2} \mathrm{O}_{4}\right)$ was studied by X-ray diffraction as a function of pressure. Pressure is shown to induce an irreversible rhombohedral to orthorhombic transition leading to a supercell determined by the combination of electron and synchrotron X-ray diffraction. This new configuration is proposed to be charge-ordered in agreement with the results of resistivity measurements.
\end{abstract}

PACS numbers: 7

Coupled phenomena between ferroelectric and ferromagnetic properties are gaining increasing interest from both a fundamental and technological point of view [1]. Although these materials are rare, the mechanisms giving rise to multiferroicity are shown to originate from particular balance between charge-spin-orbital and lattice parameters [2]. Lutetium ferrite $\left(\mathrm{LuFe}_{2} \mathrm{O}_{4}\right)$ appears as an ideal candidate to study as its multiferroic properties were shown to be directly linked to electronically-driven ferroelectricity [3]. At room temperature, the structure exhibits charge ordering [3] and is thus more complex than the usual description, i.e. the stacking along $c$ of triangular lattices of lutetium, iron and oxygen [4] (Figure 1a). Owing to the ordered charge distribution, each iron bi-layer has a dipole moment and the system is ferroelectric below the charge-ordering temperature $\left(T_{C O} \approx\right.$ $330 \mathrm{~K}$ ), which was determined based on Mössbauer spectroscopy [5], neutron [6] and electron diffraction [7]. The exact full three-dimensional charge configuration, determining the net electric polarisation of the material, is however under debate from a theoretical [8] and experimental $[6,7]$ point of view. Additionally, upon cooling, ferrimagnetism is observed below $240 \mathrm{~K}$ giving rise to particular coupling of the sequential charge ordering and spins $[9,10]$. Further interesting properties linked with spin-charge order coupling have recently been reported such as magnetoelectric response [11], giant magnetic coercitivity [10] and strong insulating behavior [5] for this material. The supercell detected by neutron diffraction is reported to have a charge ordered structure in the $a b$ plane along the [110] direction [6]. Single crystal Xray scattering studies proposed an antiferroelectric stacking of the charge order associated with an incommensurate propagation close to $\left(\frac{1}{3} \frac{1}{3} \frac{3}{2}\right)$, while diffuse scattering at $360 \mathrm{~K}$ with $\left(\frac{1}{3} \frac{1}{3} 0\right)$ propagation indicated ferroelectric short-range correlations between neighboring double layers [12].
As the electric polarization arises from the special charge order of $\mathrm{Fe}^{2+}$ and $\mathrm{Fe}^{3+}$, pressure can be expected to decrease the Fe-Fe distances and hence increase the electronically-driven ferroelectricity. Such a scenario has already been observed in $\mathrm{RMn}_{2} \mathrm{O}_{5}$ manganites [13] and the different high pressure effects upon $\mathrm{RMnO}_{3}[14]$ show that pressure is an appropriate parameter to better understand electronically-driven ferroelectricity. A very recent study was reported on the pressure effects on $\mathrm{LuFe}_{2} \mathrm{O}_{4}$ up to $2.5 \mathrm{GPa}$ by ac susceptibility and electronic transport measurements showing that pressure tends to suppress the long-range magnetic state [15]. In this Letter, we present a high-pressure structural study of $\mathrm{LuFe}_{2} \mathrm{O}_{4}$ by X-Ray diffraction and resistivity measurements. The recovered sample is further characterized by electron and synchrotron X-ray diffraction. We will show that higher pressure induces an irreversible phase transition to a high-pressure ordered structure with an orthorhombic supercell. This new phase is proposed to be due to a new charge ordered state.

$\mathrm{LuFe}_{2} \mathrm{O}_{4}$ was prepared by solid state reaction at high temperature, starting from a $10 \mathrm{~g}$ mixture of $0.485 \mathrm{Lu}_{2} \mathrm{O}_{3}$ : $0.815 \mathrm{Fe}_{2} \mathrm{O}_{3}: 0.37 \mathrm{Fe}$, pressed in the shape of a rod $(6 \mathrm{~mm}$ diameter and several cm length) and heated at $1180^{\circ} \mathrm{C}$ for $12 \mathrm{~h}$ in an evacuated silica ampoule. Neutron powder diffraction (NPD) data (recorded on 3T2 at the Laboratoire Léon Brillouin at room temperature) show that the sample is free of impurities and has the expected oxygen stoichiometry within the accuracy of the refinement. The refined cell parameters are $a_{R \overline{3} m}=3.44051(2) \AA$ and $c_{R \overline{3} m}=25.2389(2) \AA$ in the $R \overline{3} m$ space group (hexagonal setting). Measured Néel and charge ordering transition temperatures occur at $\cong 240 \mathrm{~K}[9,10]$ and $\cong 330 \mathrm{~K}[5,7]$, respectively in agreement with the literature.

Angle-dispersive X-ray diffraction data at high pressure were obtained using a laboratory source and a diamond anvil cell (DAC). The $\mathrm{LuFe}_{2} \mathrm{O}_{4}$ powder was placed 


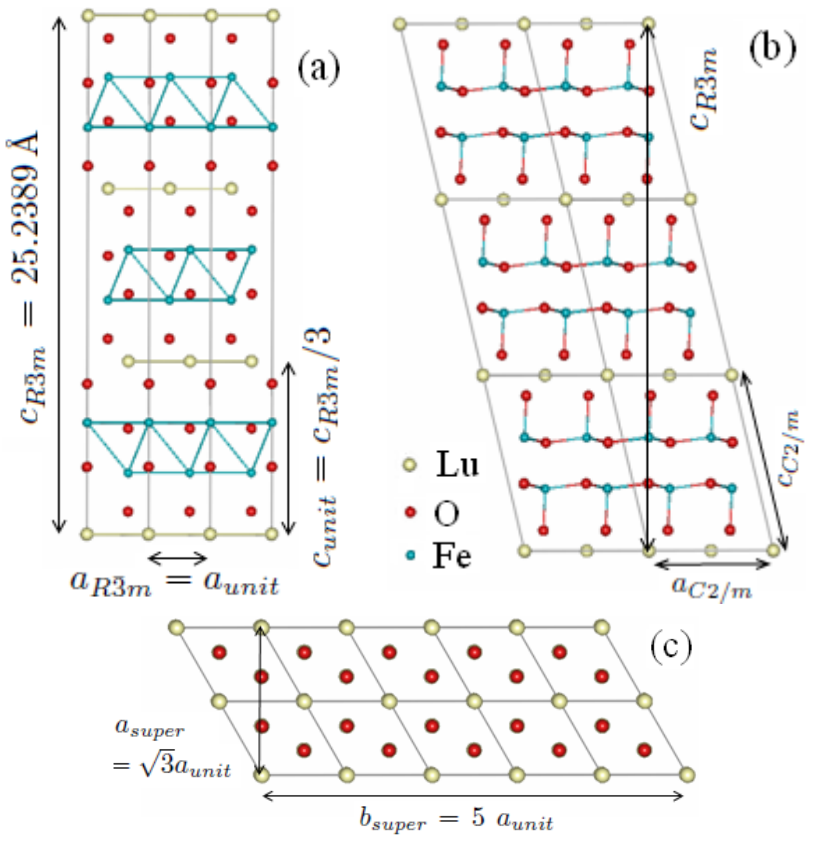

FIG. 1. a) (color online) Rhombohedral crystal structure of $\mathrm{LuFe}_{2} \mathrm{O}_{4}$. b) Equivalent monoclinic unit-cell of $\mathrm{LuFe}_{2} \mathrm{O}_{4}$ $C 2 / m$ with $a_{C 2 / m}=5.957 \AA\left[=a_{R \overline{3} m} \sqrt{3}\right], b_{C 2 / m}=3.436 \AA$ $\left[=a_{R \overline{3} m}\right]$ and $c_{C 2 / m}=8.641 \AA\left[\approx c_{R \overline{3} m} / 3\right]$ with $\beta_{C 2 / m}=$ $103.2^{\circ}$. c) Orthorhombic high-pressure modulated supercell: $a_{\text {super }} \approx \sqrt{3} a_{\text {unit }}, b_{\text {super }} \approx 5 a_{\text {unit }}$ and $c_{\text {super }} \approx 8 c_{\text {unit }}$.

in a tungsten gasket along with $\mathrm{NaCl}$ as pressure calibrant. A 21:4:1 (volume ratio methanol:ethanol: $\mathrm{H}_{2} \mathrm{O}$ ) mixture was introduced as a pressure-transmitting medium. X-ray diffraction patterns were obtained with Zr-filtered Mo radiation from a $800-\mathrm{W}$ microfocus tube. X-ray capillary optics were used, giving a $100-\mu \mathrm{m}$ diameter beam. Detection was performed with an imaging plate. Exposure times were typically between 24 and 48 hours. The intensities obtained on an imaging plate were integrated as a function of $2 \theta$ using FIT2D in order to give conventional, one dimensional diffraction profiles [16]. Pressures were measured based on equation of state of $\mathrm{NaCl}$ [17]. Cell parameters were obtained either by Le Bail method or Rietveld refinement using the software Fullprof [18]. Pressure - volume data were fitted to the Birch-Murnaghan equation of state (with $B_{0}^{\prime}$ =4) [19] . An X-ray diffraction pattern of the recovered sample in the gasket was obtained at the European Synchrotron Radiation Facility on beamline ID27, using $0.3738 \AA$ photons and a Bruker smart CCD 6500. This sample was then studied by electron microscopy, using a JEOL 200CX electron microscope.

Sample resistance was determined in a large volume multianvil press [20]. A pressed pellet of $\mathrm{LuFe}_{2} \mathrm{O}_{4}$ was placed in a pressure medium consisting of semi-sintered $\mathrm{Cr}$-doped $\mathrm{MgO}$. Resistance across the disc was monitored during compression up to $14 \mathrm{GPa}$.

In agreement with the NPD study, the X-ray diffrac-
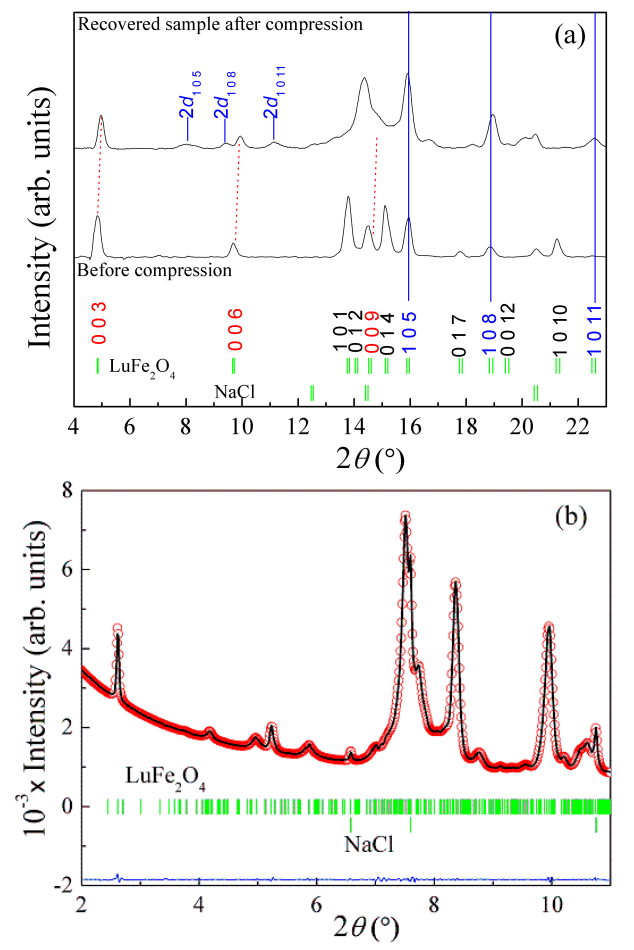

FIG. 2. (color online) a) X-ray diffraction pattern of $\mathrm{LuFe}_{2} \mathrm{O}_{4}$ at $298 \mathrm{~K}$ before compression (bottom) and of the recovered sample after compression (top). Note that the contribution from the diamond anvil cell was subtracted for clarity. Vertical ticks indicate, from the top down, the calculated positions of the reflections from $\mathrm{LuFe}_{2} \mathrm{O}_{4}$ and $\mathrm{NaCl}$. b) Le Bail fit to the $\mathrm{X}$-ray diffraction pattern of $\mathrm{LuFe}_{2} \mathrm{O}_{4}$ after compression to 12 GPa recovered at atmospheric compression pressure obtained at the ESRF (ID27). In this fit, an arbitrary orthorhombic space group with no systematic absences and a modulation vector $=\mathrm{ra}^{*}+\mathrm{sc}^{*}$ (with $\mathrm{r}=1$ and $\mathrm{s}=1 / 2$ ) were used.

tion pattern of $\mathrm{LuFe}_{2} \mathrm{O}_{4}$ at ambient conditions is consistent with structural data obtained in the literature, Figure 2a (bottom). The hexagonal unit cell parameters could be easily refined up to a pressure of $4.5 \mathrm{GPa}$ beyond which a phase mixture is observed. Above $8 \mathrm{GPa}$, the initial low-pressure form had almost entirely disappeared. Additional compression steps were made up to 12 GPa to obtain the pure high-pressure phase. The highpressure phase can be recovered at atmospheric pressure, Figure $2 \mathrm{~b}$. The diffraction pattern of the recovered, highpressure phase of $\mathrm{LuFe}_{2} \mathrm{O}_{4}$ is more complex than that of the low-pressure starting material; however, there are evident similarities between the two patterns, Figure 2 . A series of sharp reflections are observed systematically shifted to higher $2 \theta$ with respect to the $00 l(l=3 \mathrm{n})$ reflections of the low pressure form. These shifts correspond to a decrease in the $c$ lattice parameter by $2.8 \%$. A series of additional, broad low angle reflections are observed in the high-pressure pattern indicating an increase in the size of the unit cell, Figure 2. These new broad peaks are observed at close to twice the $d$-spacing of a series 
of $10 l(l=5,8,11)$ reflections of the starting material, which appear at close to same $2 \theta$ values in the recovered high-pressure form.
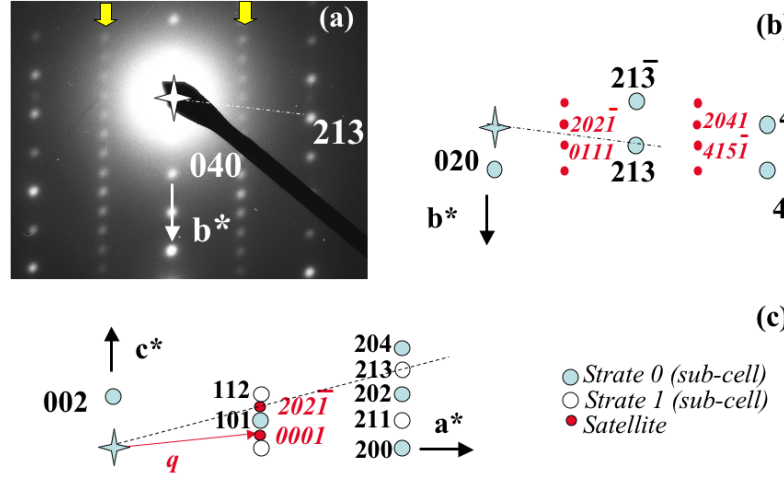

FIG. 3. (color online) a) [30 $\overline{2}]$ electron diffraction pattern of $\mathrm{LuFe}_{2} \mathrm{O}_{4}$. Only the reflections of the sub-cell are indexed (white numbers): all the satellites are aligned along the intermediate rows indicated by yellow arrows. b) Indexation of the satellites (red numbers in italics), using four indices $\mathrm{hklm}$. c) Reconstitution of [010] pattern, obtained through the rotations, which allows to evidence the modulation vector $=\mathrm{ra}^{*}$ $+\mathrm{sc}^{*}$ (with $\mathrm{r}=1$ and $\mathrm{s}=1 / 2$ ).

The transmission electron microscopy (TEM) observations of this new $\mathrm{LuFe}_{2} \mathrm{O}_{4}$ structure evidenced a mica-like morphology of the crystallites, with lamellae perpendicular to the $\vec{c}$ axis. The coupled selected area electron diffraction (SAED) and energy dispersive spectroscopy analyses were carried out in order to study the crystallinity and homogeneity of the sample. Note that some of the grains are not suitable for the investigation of reciprocal space, due to a strong misorientation of the lamellae by rotation around $\vec{c}$. The reconstruction of reciprocal space was carried out by tilting around the $\overrightarrow{\mathrm{a}^{*}}$ and $\overrightarrow{b^{*}}$ axes on the other well crystallized grains. This study shows that two systems of reflections coexist: intense spots are associated with a sub-cell and weaker, but sharp, reflections with satellites. The important changes correspond firstly with the loss of the rhombohedral symmetry of the initial $R \overline{3} m$ cell and, secondly, with the important variations in the cell parameters, both being the signatures of major structural rearrangements. The subcell parameters are the following: $\mathrm{a}_{\text {sub }} \approx 5.4 \AA, \mathrm{b}_{\text {sub }} \approx$ $17.6 \AA$ and $\mathrm{c}_{\text {sub }} \approx 32 \AA$, with $\alpha, \beta, \gamma \approx 90^{\circ}$ and the conditions limiting the reflections are $h k l: h+k+l=2 n$. However, these conditions are violated by the existence of satellite reflections, that can be all indexed considering a commensurate modulation vector $\vec{q}=1 \overrightarrow{\mathrm{a}^{*}}+1 / 2 \overrightarrow{\mathrm{c}^{*}}$, as illustrated by the $[30 \overline{2}]$ electron diffraction pattern in Figure 3. Only the reflections of the sub-cell are indexed (white numbers): all the satellites are aligned along the intermediate rows indicated by yellow arrows. The indexation of the satellites, using four indices $h k l m$, is specified in Figure 3b (red numbers in italics). The reconstituted
[010] pattern, obtained through the rotations and which allows to evidence the modulation vector $=\mathrm{ra}^{*}+\mathrm{sc}^{*}$ (with $\mathrm{r}=1$ and $\mathrm{s}=1 / 2$ ) is given in Figure 3c. The blue spots represent the $h 0 l$ reflections and the white ones the h1l reflections (located in the layer 1 ) of the sub-cell; in order to simplify the scheme, only a part of the satellites h1lm are numbered (in red), because the others overlap the reflections of the sub-cell. The new phase can thus be described by a modulated structure: $\mathrm{a}_{s u b} \approx 5.4 \AA$, $\mathrm{b}_{\text {sub }} \approx 17.6 \AA$ and $\mathrm{c}_{\text {sub }} \approx 32 \AA$, with $\alpha, \beta, \gamma \approx 90^{\circ}$ and $\vec{q}=1 \overrightarrow{\mathrm{a}^{*}}+1 / 2 \overrightarrow{\mathrm{c}^{*}}$. Because of the commensurability of the modulation vector, the high-pressure phase can be described in a 3D lattice with $a \approx 5.4 \AA, b \approx 17.6 \AA$ and $c \approx 64 \AA$, and $\alpha, \beta, \gamma \approx 90^{\circ}$.

The parameters of the sub-cell are consistent with those obtained from Le Bail refinement of the synchrotron X-ray diffraction data (Figure 2b): $a_{h p}=$ 5.4060(5) $\AA, b_{h p}=17.4570(12) \AA, c_{h p}=32.8064(25) \AA$. If the relationship between the $a$ parameters (in high and ambient pressure phases) is evident $\left[a \approx a_{R \overline{3} m} \sqrt{3}\right]$, the others are more complex. It could be easier by considering the following monoclinic unit cell $C 2 / m$ with $a_{C 2 / m}$ $=5.957 \AA\left[=a_{R \overline{3} m} \sqrt{3}\right], b_{C 2 / m}=3.436 \AA\left[=a_{R \overline{3} m}\right]$ and $c_{C 2 / m}=8.641 \AA\left[\approx c_{R \overline{3} m} / 3\right]$ with $\beta_{C 2 / m}=103.2^{\circ}$ (Figure 1b). The cell parameters of the modulated supercell are thus: $a_{\text {super }} \approx \sqrt{3} a_{\text {unit }}, b_{\text {super }} \approx 5 a_{\text {unit }}$ and $c_{\text {super }} \approx$ $8 c_{\text {unit }}$ (Figure 1c). The (001) plane is obtained from that considered in a hexagonal setting, with a larger surface and a strong distortion $5 a_{\text {super }} / b_{\text {super }} \approx 1.55$ instead of $\sqrt{3}$. The five-fold multiplicity of the $b_{\text {super }}$ parameter is clearly observed in the ED pattern shown in Figure 3 a. The value of the $c$ parameter, $8 c_{\text {unit }}$, confirms the disappearance of the rhombohedral stacking mode of the layers along this direction.

The high-pressure phase is found to be less compressible than the low-pressure phase, Figure $4 \mathrm{a}, B_{0}=$ 188(3) $\mathrm{GPa}$ and $B_{0}=148(1) \mathrm{GPa}$ respectively. The recovered sample at atmospheric pressure is only about $0.5 \%$ denser than the starting phase, which is evidence that the $\mathrm{LuO}_{1.5}$ layers and $\mathrm{Fe}_{2} \mathrm{O}_{2.5}$ layers are retained. The $\mathrm{LuO}_{1.5}$ close packing is similar to that of alumina $\left(\mathrm{Al}_{2} \mathrm{O}_{3}\right)$, which is known to be stable at high pressure [21]. Concerning the $\mathrm{Fe}_{2} \mathrm{O}_{2.5}$ bilayers if pressure induced a (partial) increase in the iron coordination number with distinct environments for $\mathrm{Fe}^{2+}$ and $\mathrm{Fe}^{3+}$, this would result in a volume change of more than $5 \%$, which is not the case. The $\mathrm{Fe}_{2} \mathrm{O}_{2.5}$ bilayers in agreement with the TEM results retain a similar network of triangular bipyramids and the increase in the size of the unit cell is only due to rearrangements. Accordingly, the initial $\mathrm{Fe}^{2+} / \mathrm{Fe}^{3+}$ charge order in $\mathrm{LuFe}_{2} \mathrm{O}_{4}$ is most probably affected and a new charge ordered structure could thus be obtained in the high-pressure form.

Therefore, the observed transition as a function of pressure is due to changes in charge ordered structure of $\mathrm{Fe}^{2+}$ 

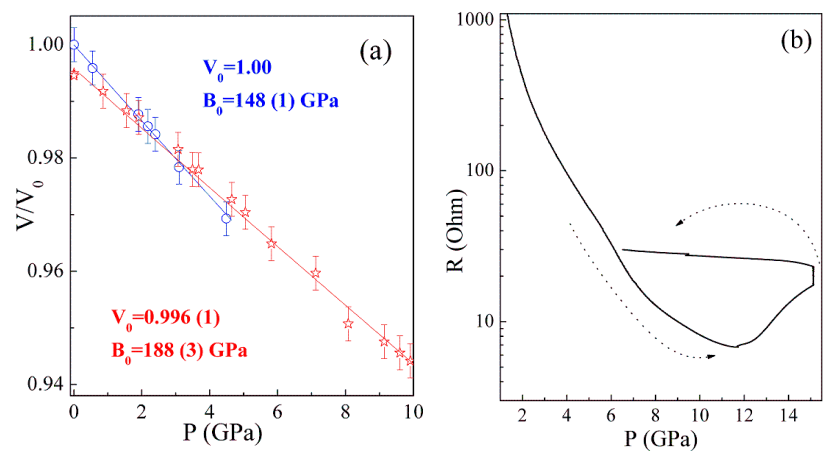

FIG. 4. (color online) a) Relative volume and b) resistance measurements of $\mathrm{LuFe}_{2} \mathrm{O}_{4}$ as a function of pressure at 298 $\mathrm{K}$. The $B_{0}$ values are calculated using the Birch-Murnaghan state equation $\left(B_{0}^{\prime}=4\right)$ [19]. Open circles and open stars indicate low-pressure and high-pressure phases (obtained upon decompression) respectively.

and $\mathrm{Fe}^{3+}$, while retaining five-fold coordination. Pressure enhanced charge ordering has already been reported in literature $[22,23] . \mathrm{As} \mathrm{Fe}^{2+}$ and $\mathrm{Fe}^{3+}$ in triangular bipyramid arrangements have different ionic radii, i.e. about $0.1 \AA$, they exhibit different bond lengths and local compressibilities. Hence, it is possible to observe a new pressure induced charge-ordered structure (less compressible) that can be recovered at atmospheric pressure. By analogy with the work of Angst et al. [12], the orthorhombic high-pressure phase obtained in our study could probably have an antiferroelectric stacking of the dipole moment which explains the large size of the supercell. In this electronically-driven ferroelectricity, such a charge arrangement is probably of greatest interest as the spontaneous polarization arises from the electronic repulsion between the two iron sites due to the decrease in the $\mathrm{Fe}^{2+}-\mathrm{Fe}^{3+}$ distance under pressure. Additionally, such an enhanced charge ordering should imply a modification of the electronic properties of $\mathrm{LuFe}_{2} \mathrm{O}_{4}$ with an increase of the resistivity as a consequence of the reduction of the electron hopping [6] between $\mathrm{Fe}^{2+}$ and $\mathrm{Fe}^{3+}$. The increase in resistivity observed above $11 \mathrm{GPa}$, Figure $4 \mathrm{~b}$, corresponds to the pure high-pressure phase and therefore supports the charge-ordered character of this new structure obtained with pressure. Such an increase in resistivity could also be of particular interest to polarize $\mathrm{LuFe}_{2} \mathrm{O}_{4}$ as it is known that polarization-electric field hysteresis loop could not be obtained due to current leakage at atmospheric pressure [3].

In conclusion, our results show that pressure induces a new charge order in $\mathrm{LuFe}_{2} \mathrm{O}_{4}$, which is recoverable at atmospheric pressure. In accordance with the new large orthorhombic supercell and the conservation of the five fold coordination, the strong X-ray superlattice reflections arise from distinct $\mathrm{Fe}$ environments with character- istic Fe-O distances. This work should motivate further studies on the high-pressure form (dielectric, polarization, magnetic properties).

The authors wish to thank Gaston Garbarino from the beamline ID27 of the European Synchrotron Radiation Facilities (ESRF). Financial support for this work was partially provided by the French Agence Nationale de la Recherche, Grant N ${ }^{\circ}$ ANR-08-BLAN-0005-01 and $N^{\circ}$ JC08-331297. The multi-anvil apparatus of Laboratoire Magmas et Volcans is financially supported by the Centre National de la Recherche Scientifique (Instrument National de l'INSU).

* jerome.rouquette@univ-montp2.fr

[1] S. W. Cheong and M. Mostovoy, Nat. Mat. 6, 13 (2007).

[2] D. V. Efremov, J. van den Brink, and D. I. Khomskii, Nat. Mater. 3, 853 (2004).

[3] N. Ikeda et al., Nature (London) 436, 1136 (2005).

[4] M. Isobe, N. Kimizuka, J. Iida, and S. Takekawa, Acta Cryst. C 46, 1917 (1990).

[5] X. S. Xu et al., Phys. Rev. Lett. 101, 227602 (2008).

[6] Y. Yamada, K. Kitsuda, S. Nohdo, and N. Ikeda, Phys. Rev. B 62, 12167 (2000).

[7] Y. Zhang, H. X. Yang, C. Ma, H. F. Tian, and J. Q. Li, Phys. Rev. Lett. 98, 247602 (2007).

[8] H. J. Xiang and M.-H. Whangbo, Phys. Rev. Lett. 98, 246403 (2007).

[9] J. Iida et al., J. Phys. Soc. Jpn. 62, 1723 (1993).

[10] A. D. Christianson et al., Phys. Rev. Lett. 100, 107601 (2008).

[11] M. A. Subramanian et al., Adv. Mater. 18, 1737 (2006).

[12] M. Angst et al., Phys. Rev. Lett. 101, 227601 (2008).

[13] C. R. dela Cruz, B. Lorenz, Y. Y. Sun, Y. Wang, S. Park, S-W. Cheong, M. M. Gospodinov, and C. W. Chu, Phys. Rev. B 76, 174106 (2007).

[14] K. Noda, S. Nakamura, J. Nagayama, and H. Kuwahara, J. Appl. Phys. 97, 10C103 (2005).

[15] X. Shen, C. H. Xu, C. H. Li, Y. Zhang, Q. Zhao, H. X. Yang, Y. Sun, J. Q. Li, Q. Jin, and R. C. Yu, Appl. Phys. Lett. 96, 102909 (2010).

[16] A. P. Hammersley, S. O. Svensson, M. Hanfland, A. N. Fitch, and D. Hausermann, High Press. Res. 14, 235 (1996).

[17] F. Birch, J. Geophys. Res. 91(B5), 4949 (1986).

[18] J. Rodriguez-Carvajal, Physica B. 192, 55 (1993).

[19] F. D. Murnaghan, Proc. Natl. Acad. Sci. U.S.A. 30, 244 (1944); F. Birch, Phys. Rev. 71, 809 (1947).

[20] T. Hammouda, Earth Planet. Sci. Lett. 214, 357 (2003).

[21] A. P. Jephcoat, R. J. Hemley, and H. K. Mao, Physica B 150, (1988).

[22] L. Pinsard-Gaudart, N. Dragoe, P. Lagarde, A. M. Flank, J. P. Itie, A. Congeduti, P. Roy, S. Niitaka, and H. Takagi, Phys. Rev. B 76, 045119 (2007).

[23] W. Zhang, L. D. Yao, L. X. Yang, Z. X. Liu, C. Q. Jin, and R. C. Yu, J. Appl. Phys. 107, 023914 (2010). 